\section{CASE REPORT}

I.A. Kaminsky

A.-M. Wang

J. Olsen

S. Schechter

J. Wilson

R. Olson

\title{
Central Nervous System Crystal-Storing Histiocytosis: Neuroimaging, Neuropathology, and Literature Review
}

SUMMARY: CSH is a very uncommon lesion and is distinctly unusual in the brain. We report a case of $\mathrm{CSH}$ within the brain parenchyma in a 27-year-old woman with Crohn disease. Advanced radiologic imaging and anatomic pathology correlation allow this report to serve as a reference for future similar cases.

ABBREVIATIONS: $\mathrm{A}=$ amplitude; $\mathrm{Cho}=$ choline; $\mathrm{Cr}=$ creatine; $\mathrm{Cr} 2=$ secondary creatine; $\mathrm{CSH}=$ crystal-storing histiocytosis; FLAIR = fluid-attenuated inversion recovery; MTT = mean transit time; $\mathrm{NAA}=\mathrm{N}$-acetylaspartate; $\mathrm{P}=$ peak TTP $=$ time to peak

C $\mathrm{SH}$ is an unusual entity. When previously reported, $\mathrm{CSH}$ was seen in the setting of abnormal expression of monoclonal immunoglobulins. ${ }^{1}$ These apparent intralysosomal inclusions of crystalline aggregates can present diagnostic difficulties whether they occur in the bone marrow or less commonly in extramedullary sites. Involvement of the brain parenchyma has not been previously reported, to the best of our knowledge. We present the case of a patient with left-sided neurologic symptoms and a positive radiologic and anatomic pathology work-up.

\section{Case Report}

A 27-year-old woman was diagnosed with Crohn disease in July 2008. In October of the same year, she was hospitalized for an exacerbation of her Crohn disease. On approximately day 6 of her admission, she developed a left-sided tremor and reported numbness and a tingly sensation on her left side. She denied any clear seizure activity. A diagnostic work-up was conducted, including head CT and brain MR imaging with MR perfusion, diffusion, and spectroscopy. A brain biopsy was also performed.

The initial head CT scan showed slightly increased attenuation to the white matter, with subtle enhancement in the right centrum semiovale. Subsequent MR imaging (Fig 1) demonstrated a 6.1-cm area of abnormal signal intensity (slightly decreased T1 signal intensity and moderately increased T2 signal intensity) in the same region with surrounding vasogenic edema. Minimal mass effect was seen. FLAIR images (Fig 2) demonstrated numerous flow-void-like areas, which were associated with the foci of post-gadolinium enhancement. MR spectroscopy (TE, $135 \mathrm{~ms}$ ) showed elevation of the choline peak, a decreased NAA peak, and the presence of lactate (Fig 3). MR perfusion (Fig 4) demonstrated slightly increased regional cerebral blood volume and regional cerebral blood flow with decreased MTT and TTP of the lesion. Differential considerations based on these imaging findings are nonspecific and include a perivascular inflammatory process, white matter disease, and granulomatous or neoplastic processes.

Received August 31, 2009; accepted after revision September 16.

From the Departments of Diagnostic Radiology (I.A.K.), Neuroradiology (A.-M.W.), Neurology (S.S.), Pathology (J.W.), and Neurosurgery (R.O.), William Beaumont Hospital, Royal Oak, Michigan; and Wayne State University School of Medicine (J.O.), Detroit, Michigan.

Please address correspondence to lan A. Kaminsky, MD, William Beaumont Hospital, 3601 W 13 Mile Rd., Royal Oak, Ml 48073; e-mail: ian.kaminsky@beaumont.edu

DOI 10.3174/ajnr.A1983

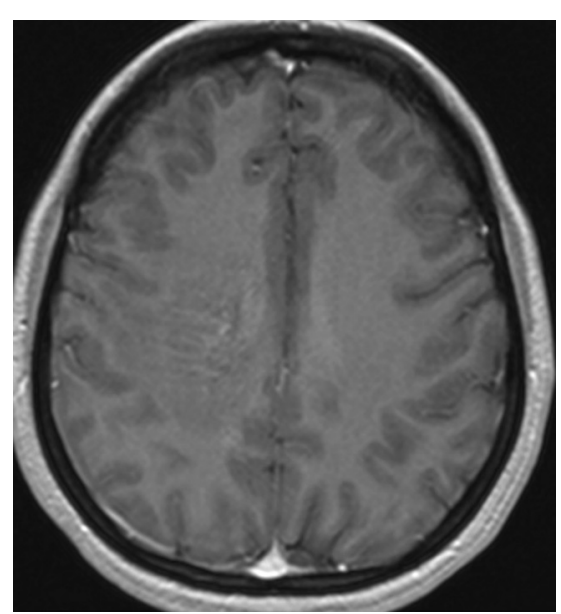

Fig 1. Axial T1-weighted image with intravenous administration of gadolinium contrast. Serpentine contrast enhancement is seen in the right centrum semiovale.

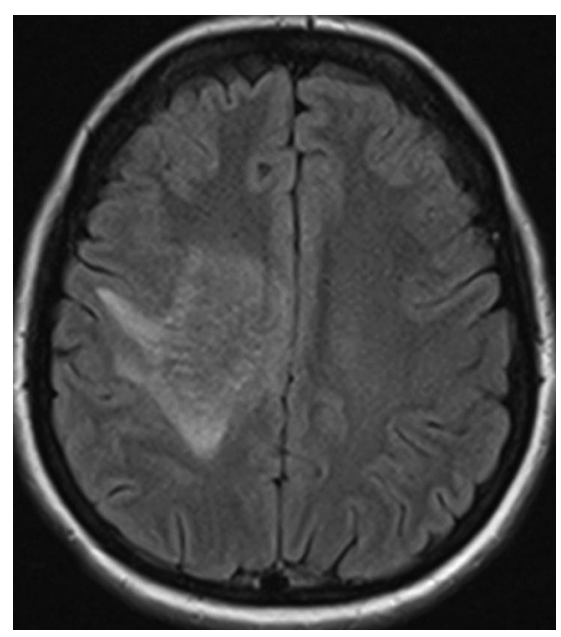

Fig 2. Axial FLAIR image shows abnormal increase of signal intensity in the centrum semiovale on the right with serpentine low signal intensity within it. No significant mass effect is seen.

Pathologic examination revealed unusual intracytoplasmic inclusions in the macrophages, confirmed with CD68 antibody stain. There was associated mild perivascular chronic inflammation and some focal chronic inflammation in the vessel walls. Hematoxylineosin staining (Fig 5) revealed that the material was eosinophilic with 


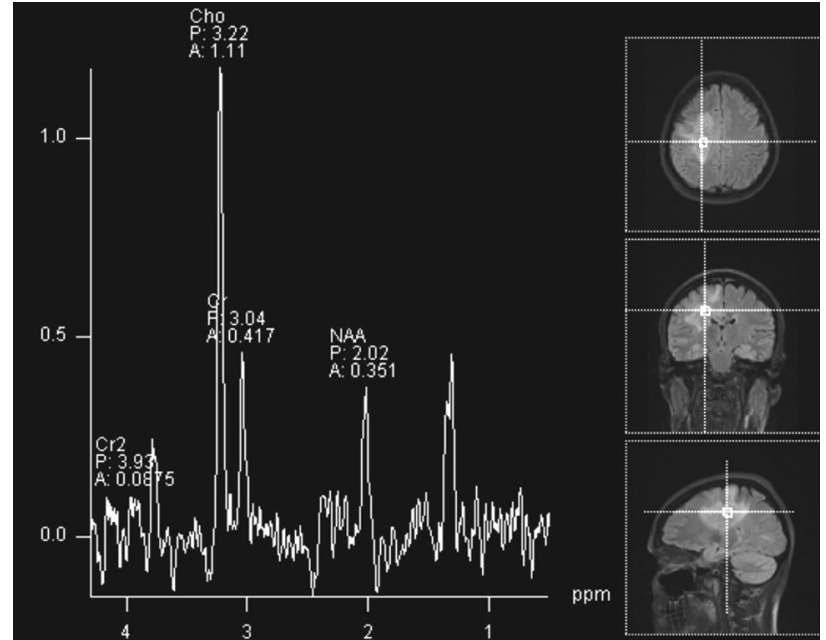

Fig 3. Proton MR spectroscopy of a lesion in the right centrum semiovale shows elevation of the choline peak and decrease of the NAA peak. There is also lactate at $1.3 \mathrm{ppm}$

a finely striated appearance. The affected macrophages were most prominent in a perivascular distribution, formed perivascular cuffs, and were present within the parenchyma of deeper biopsies. The deeper biopsies demonstrated reactive astrocytosis, without definite inclusions in the astrocytes. The inclusions within macrophages were magenta-colored on Diff-Quick-stained frozen tissue. There was evidence supporting the preservation of myelin and neurons/axons. Electron microscopy (Fig 6) revealed that the inclusions consisted of rigid finely lamellar osmophilic material, likely crystalline in nature. The material was arranged in needlelike bundles, as well as in thicker bundles. In some sections, the bundles seem to have been located within membrane-bound spaces, favoring lysosomes. These pathologic changes are those of a non-neoplastic reactive/inflammatory process and are consistent with the diagnosis of CSH.

\section{Discussion}

$\mathrm{CSH}$ is a very uncommon lesion and is distinctly unusual in the brain. This case illustrates advanced imaging findings of this rare process. We believe that the slightly increased regional cerebral blood volume and increased regional cerebral

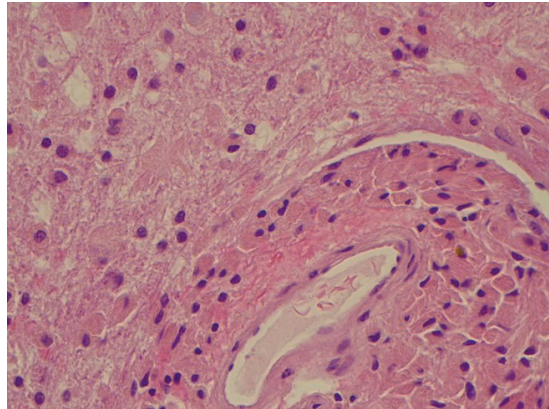

Fig 5. Photomicrograph of deeper tissue shows inclusion-containing histiocytes in a perivascular and parenchymal distribution (hematoxylin-eosin, medium power; $200 \times$ magnification)

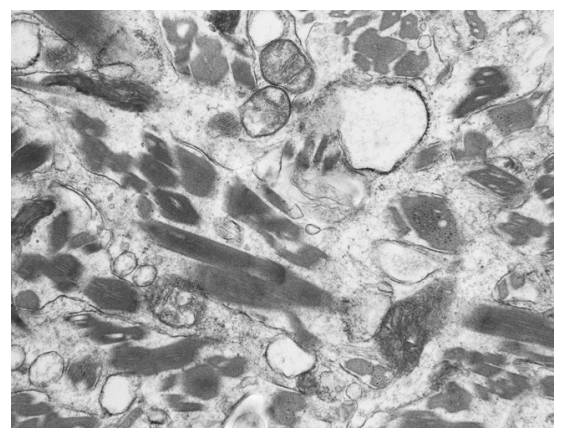

Fig 6. Electron microscopy, ultrastructural photomicrograph shows abundant moderately osmophilic needlelike inclusions that appear geometrically irregular-to-rhomboid-shaped in cross-section within histiocyte cytoplasm. The inclusions show regular fine linear periodicity and are noted to occasionally be within phagolysosomes. No inclusions are seen within the mitochondria.

blood flow are related to hyperemia from the underlying reactive/inflammatory process. Additionally, we postulate that the flow-void-like areas on FLAIR imaging represent prominent veins, which result from the partial perivascular nature of the inflammation. Mild postcontrast enhancement likely relates to an element of breakdown at the blood-brain barrier from the same process. NAA is a marker of neuronal cell health. Decreased NAA peak is commonly seen in nonspecific neuronal loss, including ischemia, trauma, inflammation, infection,

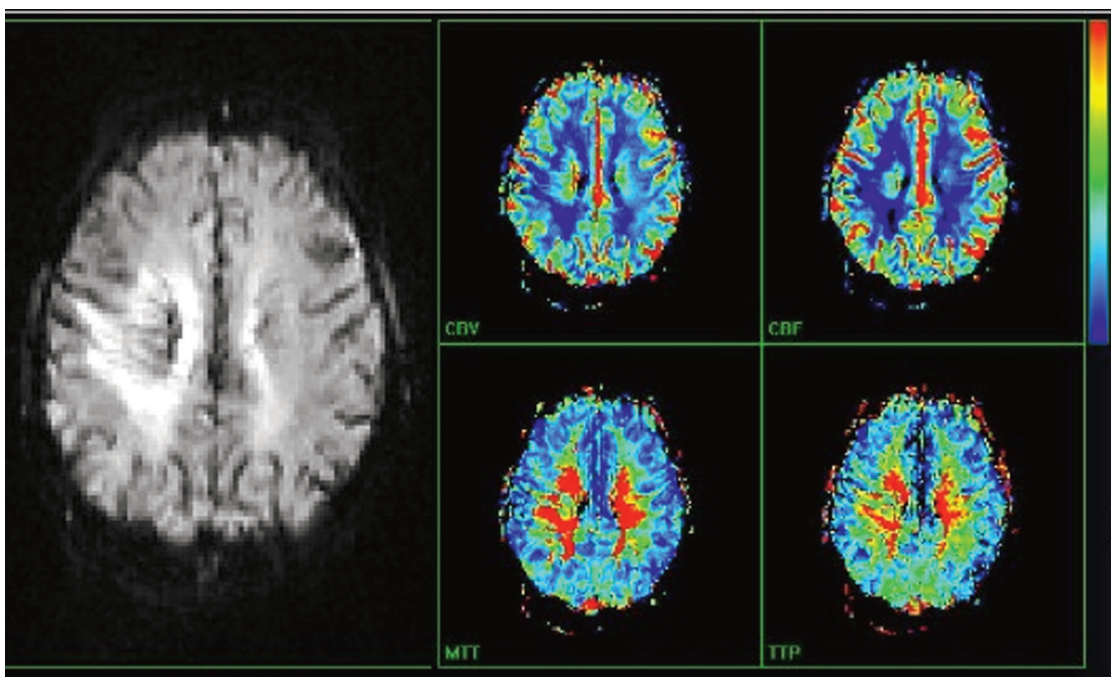

Fig 4. Contrast-enhanced MR perfusion image shows a slight increase of regional cerebral blood volume and regional cerebral blood flow. There is also decreased MTT and TTP of the lesion. 
tumors, dementia, and gliosis. Choline is a marker of membrane turnover and is present to a greater degree in white matter than in gray matter. Choline is increased in tumors, inflammation, and chronic hypoxia. Lactate is not seen in the normal brain. It is the end product of anaerobic respiration and is elevated in foamy macrophages. The presence of lactate can be seen in patients with ischemia, inborn errors of metabolism, tumors, abscess, and inflammation. In our case, the constellation of MR spectroscopy findings could be related to inflammation.

In previous case reports, morphologically similar crystalline material was most often comprised of immunoglobulin in the setting of monoclonal gammopathy. ${ }^{1}$ However, cases associated with Charcot-Leyden crystals related to eosinophilic inflammation, resulting from treatment with the drug clofazimine, have also been reported. ${ }^{2,3}$ Our pathologist raised the latter possibility because the patient's tissue sample had a somewhat orange discoloration, which can be seen after treatment with clofazimine. However, research indicates that this antimycobacterial medication does not cross the blood-brain barrier. An extensive Web-based literature search for other etiologies failed to yield prior reports of $\mathrm{CSH}$ associated with Pentasa-type (mesalamine) medications (the patient had a history of noncompliant treatment with Pentasa). However, the search did yield 1 prior case report in 1984 of a 38 -year-old woman with "crystalline encephalopathy."
Our patient's brain biopsy did demonstrate the focal presence of perivascular lymphocytes and lymphocytes focally within the vessel wall. The previously described features may suggest angiitis, ${ }^{5}$ but the possibility that the inflammatory cells merely represent an inflammatory response to tissue injury from whatever is causing the crystalline material to accumulate cannot be excluded. Previous cases with accompanying advanced radiologic imaging of such a disease process within the brain parenchyma have not been identified during our extensive Web-based literature search.

\section{References}

1. Lebeau A, Zeindl-Eberhart E, Müller EC, et al. Generalized crystal-storing histiocytosis associated with monoclonal gammopathy: molecular analysis of a disorder with rapid clinical course and review of the literature. Blood 2002;100:1817-27

2. Sukpanichnant S, Hargrove NS, Kachintorn U, et al. Clofazimine-induced crystal-storing histiocytosis producing chronic abdominal pain in a leprosy patient. Am J Surg Pathol 2000;24:129-35

3. Lewis JT, Candelora JN, Hogan RB, et al. Crystal-storing histiocytosis due to massive accumulation of Charcot-Leyden crystals: a unique association producing colonic polyposis in a 78-year-old woman with eosinophilic colitis. Am J Surg Pathol 2007;31:481-85

4. Pezeshkpour G, Stuart TD, Estridge MN, et al. Crystalline encephalopathy: cerebral immunoprotein deposits and isolated angiitis. Ann Neurol 1984;17:96-99

5. Molloy ES, Singhal AB, Calabrese LH. Tumor-like mass lesion: an under-recognized presentation of primary angiitis of the central nervous system. Ann Rhem Dis 2008;67:1732-35. Epub 2008 Jul 14 